Unfallchirurg 2016 $\cdot 119: 68$

DOI 10.1007/s00113-015-0103-y

Online publiziert: 23. November 2015

(c) Springer-Verlag Berlin Heidelberg 2015

CrossMark
F. von Matthey ${ }^{1}$ K.F. Braun ${ }^{1} \cdot$ M. Hanschen ${ }^{1} \cdot$ F. Pohlig ${ }^{1}$ E.C. Schubert ${ }^{2}$. E. Matevossian ${ }^{3} \cdot$ P. Hoppmann ${ }^{4} \cdot$ K.-G. Kanz ${ }^{1} \cdot$ P. Biberthaler ${ }^{1}$

${ }^{1}$ Klinik und Poliklinik für Unfallchirurgie, Klinikum rechts der Isar der Technischen Universität München, München, Deutschland

${ }^{2}$ Institut für diagnostische und interventionelle Radiologie, Klinikum rechts der Isar der Technischen Universität München, München, Deutschland

${ }^{3}$ Chirurgische Klinik und Poliklinik, Klinikum rechts der Isar der Technischen Universität München, München, Deutschland

${ }^{4}$ I. Medizinischen Klinik und Poliklinik, Klinikum rechts der Isar der Technischen Universität München, München, Deutschland

\section{Erratum zu: Zustand nach Reanimation}

\section{Eine Indikation für die „Traumaspirale“?}

\section{Erratum zu: \\ Unfallchirurg 2015}

DOI 10.1007/s00113-015-0045-4

In der Online-Version dieses Beitrags wurde der Beitragstitel zunächst falsch übersetzt. Der englische Titel lautet korrekt: „Cardiac post-resuscitation care. An indication for trauma whole-body CT?"

Wir bitten den richtigen Titel zu beachten und entschuldigen uns für den Fehler.

Die Redaktion

\section{Korrespondenzadresse}

\section{F. von Matthey}

Klinik und Poliklinik für Unfallchirurgie,

Klinikum rechts der Isar der Technischen

Universität München

Ismaningerstraße 22, 81675 München

VonMatthey@uchir.me.tum.de 